\title{
GPPS-CH-2020-0151
}

\section{NON-AXISYMMETRIC DESIGN-OPTIMIZATION OF INLET GUIDE VANES FOR A FAN WITH INLET DISTORTION}

\author{
Sandeep Kumar \\ Mark G. Turner \\ Gas Turbine Simulation Lab \\ University of Cincinnati \\ kumarsp@mail.uc.edu \\ mark.turner@uc.edu \\ Cincinnati, Ohio, USA
}

\author{
Mark L. Celestina \\ NASA Glenn Research Centre \\ mark.celestina@nasa.gov \\ Cleveland, Ohio, USA
}

\begin{abstract}
For tail-mounted thrusters, Inlet Guide Vanes (IGVs) are required to support the nacelle, and to improve fan performance under complex conditions imposed by Boundary Layer Ingestion (BLI). This paper presents the optimization of an IGV row to demonstrate a novel non-axisymmetric 3D blade design framework with focus on its parametrization scheme, and multi-fidelity system for design of typical distortion tolerant blade row(s). A key feature of this design system is the capability to produce and optimize spanwise $(r)$ and circumferentially $(\theta)$ non-axisymmetric 3D blade shapes using the harmonics-based parameters and implemented in the open source code, NAX. The purpose is to reduce the downstream rotor incidence and increase stage performance under 2D circumferential distortion due to the fuselage and vertical stabilizer. For process validation, a manual design was first created using only magnitude and phase as input for the $15 \%$ span (near-hub) location. A least squares method and finally a Genetic Algorithm (GA) was used to optimize blade shapes to best match with the clean flow design and reduce the stage losses. The results are further discussed in terms of the parameters that enable an exploration of a large design space, for general purpose multi-row non-axisymmetric turbomachinery design.
\end{abstract}

\section{INTRODUCTION}

Under the NASA Advanced Air Transport Technology (AATT) program, a key aspect of single aisle $\mathrm{N}+3$ designs is their turboelectric distribution propulsion (TeDP) architecture. It is achieved by decoupling of thrust and power elements and allows them to operate at their individual peak efficiencies (Felder et.al, 2009; Kim et.al., 2008). For general tubeand-wing single aisle subsonic configurations like STARC-ABL, a tail cone thruster (TCT) unit is used aft of the fuselage to accelerate the low momentum air in the boundary layer of the aircraft fuselage (Armstrong et al., 2012) and thus helps in reducing the overall profile drag due to the airframe.

The fuselage boundary layer and vertical tail wake results in complex flow structure interactions that severely influences the aerodynamic performance and aero-mechanical stability of a BLI fan. Swirl angle variation presented to the downstream rotor is caused by the propagation of the mass flow distortion (Longley and Greitzer, 1992; Kurzke, 2008). The regions for low axial velocity correspond to the regions of high flow angle into the rotor. Swirl angle distortion leads to a varying incidence into rotor blade sections, while the radial angle distortion effectively creates positive incidence in a meridional plane relative to the hub endwalls. In addition to the inlet distortion, transonic flow conditions also pose a unique challenge, incurring shock-boundary layer interactions. Being hyperbolic in nature (numerically), the understanding of the flow field coupling occurring at various length scales is important to understand the effect of shock structures on upstream and downstream blade rows (Greitzer et.al.,1978; Greitzer and Griswold, 1976). The analysis presented for 1 stage design (Gunn and Hall, 2017) has shown that primary source of distortion in stator inlet swirl angle is actually the circumferential mass flux, which particularly lies in the low momentum region (boundary layer). 
The TCT module discussed in this paper is a $1 / 4$ scale 1.5 stage transonic propulsor unit. BLI effects cause a severe unsteadiness on the rotor. To reduce this effect of distortion on the BLI fan, the provision of using non-axisymmetric IGVs are explored. A schematic of the TCT along with full scale dimensions and aerodynamic details are shown in Figure 1. Previous studies (Shaw et.al., 2013) have found that IGVs lead to a reduction in a clean flow performance but reduce the fan sensitivity to inlet distortion and improves its stall margin. In particular for a transonic fan, IGVs (variable or static) are primarily used to reduce the shock strength on the rotor (Leichtfuss et.al, 2012). Studies conducted by (Gorrell et.al, 2001; Gorrell et.al, 2006) have demonstrated that shock-wave interactions of IGV-Rotor leads to the rotor synchronous vortex-shedding. They also established that the shape of the vortices by the rotor depends upon the axial spacing and the loading of the IGV.

$\begin{array}{ll}\text { Cruise Mach } & 0.785 \\ \text { FPR } & 1.25 \\ \text { Mass Flow } & 10.3492\left[\mathrm{~kg} \mathrm{~s}^{-1}\right] \\ \text { Reference Pressure } & 66.49[\mathrm{kPa}] \\ \text { Reference } & 265.08[\mathrm{~K}] \\ \text { Temperature } & \\ \text { Reference Density } & 0.8689\left[\mathrm{~kg} \mathrm{~m}^{-3}\right] \\ \text { Rotor tip Mach no. } & 1.15\end{array}$

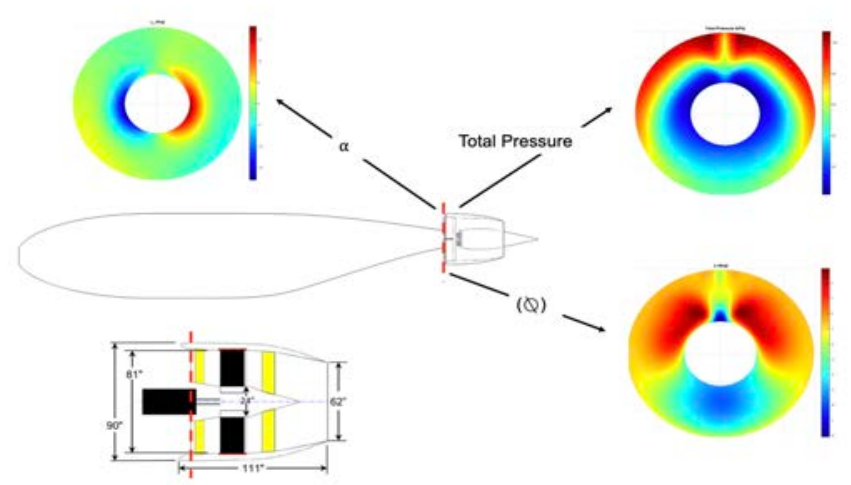

Figure 1: Schematic of TCT unit along with the boundary conditions by NASA GRC (AoA=2 degree).

In the present study, the IGV design is optimized to reduce the effects of complex 2D distortion for improved IGV wake interaction with the rotor and reduced aero-mechanical stress (steady and unsteady). The radial and circumferential profiles of total pressure, angle $(\alpha)$ and meridional angle $(\phi)$ were made available by NASA GRC (Figure 1). As per NASA/UC nomenclature, the present case name is designated as A643. The scope of this paper is limited to design and development of the 3D blade profiles and flow path of TCT unit. Within NASA/UC design teams, it was decided to develop the 1/4 scale TCT design which can be tested in a wind tunnel and analyse its performance characteristics first. The multi-fidelity design-optimization system presented in this paper uses the capability of the in-house open source code NAX (Kumar et al., 2018), for development of a distortion tolerant TCT. It uses the Fourier modes (magnitude and phase) values as input parameters for the circumferential and spanwise control $(r, \theta)$ of 3D blade shapes. The integration of NAX with multi-objective design-optimization system (Figure 2), user defined blade parameters can be optimized to achieve the aero-performance characteristics of TCT. Using the example of IGV design, the capability is demonstrated of the system to optimize the outlet flow angles to reduce the incidence variation on the rotor. The key feature of this system is the NAX parametrization scheme, which allows representation of a large design space using minimal design parameters. For complex flow fields like BLI, NAX is aerodynamically intuitive for modifying blade shapes circumferentially. As discussed in (Kumar et al. 2018) NAX is capable of producing parametric blade shapes with independent control in $(r, \theta)$ direction for blade parameters.

\section{METHODOLOGY}

\section{Development of baseline TCT:}

A multi-fidelity design-optimization system was developed to design the baseline TCT as shown in Figure 2. First, meanline/through-flow design for 1.5 stage TCT unit is developed using in-house T-AXI solver suite (Turner et.al., 2011). The T-AXI code was further modified enabling use of 1-D radial profiles of losses, blockage and normalized angular momentum as boundary conditions for through-flow calculations. The approach here is to optimize the clean flow TCT design for mass averaged 1D (radial) inlet PT (total pressure) distortion using low-fidelity tools, which can be further improved during high-fidelity 3D stage design. For this, the optimum 1.5 stage TCT from low-fidelity design is further improved at high-fidelity 3D level by optimizing it again for stage efficiency using the 1D $(r)$ total pressure profile (Mandel et al. 2020).. The IGV from this 1.5 stage 3D optimum design is used as the baseline for optimization accounting for circumferential distortion $(r, \theta)$. 


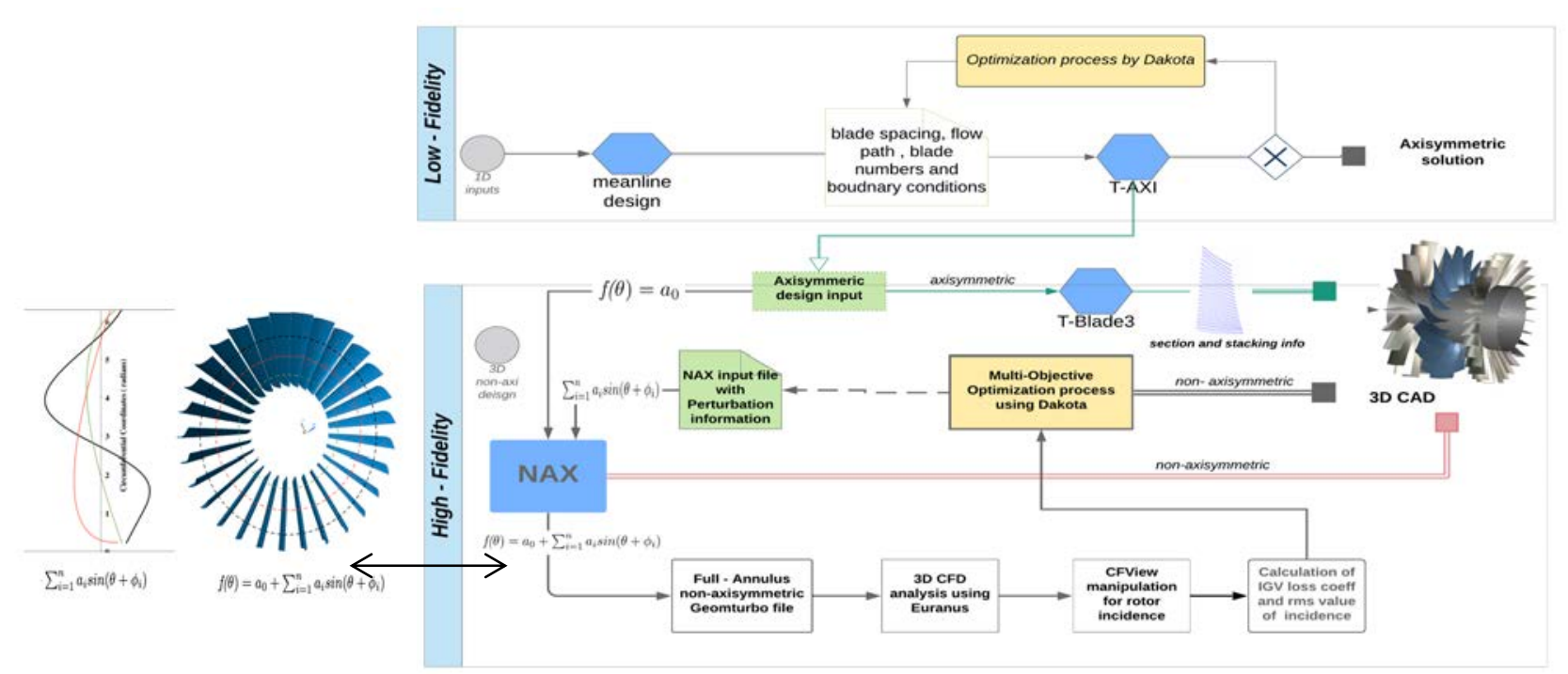

Figure 2 : Multi-fidelity design system and NAX integration for TCT development

\section{Grid Resolution Study:}

For the grid resolution study, a one-passage analysis was used with a median value of total pressure (2D distortion) (i.e., $72,300 \mathrm{~Pa})$ at the IGV inlet and $\mathrm{M}=0.58$ at exit. A total of 3 different $\mathrm{y}+$ values $(1,30$ and 50$)$ were used to calculate different wall cell heights, corresponding to fine and coarse mesh types using FINE/Turbo AutoGrid (Numeca Fine/Turbo v12.2), and to verify the use of extended wall functions with the one-equation SA turbulence model. The grid was generated with 129 spanwise elements and $(3,7,3)$ grid levels which approximates to 1.3 million nodes for one passage with structured O4H topology. The variation in IGV loss coefficient (PT1 - PT2 / PT1 - p2) (Denton,1993) was found to be $21 \%$ higher for $\mathrm{y}^{+}=1$ coarse grid against fine grid solution. For $\mathrm{y}^{+}=30$ and 50 , the loss coefficient is calculated to be $7 \%$ higher for coarse grid levels against fine grid, and within $0.25 \%$ for corresponding coarse and fine grids at these $y+$ values. This difference for different $y+$ values is because of the element size and grid spacing. With this information, we concluded that grid with $\mathrm{y}+=30$ and 50 , both have similar estimations for losses using corresponding coarse and fine grids. Hence, a computational grid with $\mathrm{y}+=50$ (coarse) was chosen with total of $6,875,700$ grid points for full annulus mesh using a $4 \%$ span interpolation to capture non-axisymmetry.

\section{NAX parametrization scheme:}

NAX is a design system used to create 3D blade shapes in a multi-row turbomachinery configuration with a combination of axisymmetric/non-axisymmetric type rows, using parametrization of blade parameters like aerodynamic sweep/ lean, inlet / exit angles, chord length and thickness distribution. NAX comprises of T-Blade3 (Siddappajji and Turner 2012; Turner, 2017; Sharma et al. 2020), and represents the blade parameters using cubic spline in the spanwise direction $(f(r))$. NAX uses the Fourier coefficients to create a smooth distribution of the blade design parameters in radial and circumferential direction $(f(r, \theta))$, thus adding another dimension to the blade design space. These design parameters can be separately controlled at independent span locations (using different number of modes), which gives it multi-mode multi-parameter capability to control design space. Using NAX, at any arbitrary span, the overall non-axisymmetric blade property is defined using its axisymmetric component $\left(a_{0}\right)$ and additional perturbations $\left(\sum_{i}^{n} a_{i} \sin \left(\theta+\emptyset_{i}\right)\right)$ ( Figure 2$)$,

$$
f(\theta)=a_{0}+\sum_{i}^{n} a_{i} \sin \left(\theta+\emptyset_{i}\right)
$$

The axisymmetric values represent the clean flow solution and the perturbations represent the departure from ideal clean flow behaviour (Figure $\underline{3}$ ). These magnitude and phase values can be assigned/modified manually or as variable by an optimizer (Dakota) to represent distortion (Adams et al. 2020). The essence and utility of NAX lies on definition of these 'perturbations' which are the magnitude and phase values (Fourier modes) of the blade parameters. An intuitive parametrization scheme efficiently represents the design space essential for development of an efficient MDO (MultiDisciplinary Optimization) system (Singh, S.K., 2017). The present paper primarily focuses on use of IGV exit angles to trace this distortion pattern on rotor incidence as relative-flow angle (Figure $\underline{3}$ ). A detailed description on the basics and working of NAX can be found in (Kumar et al. 2018), and documentation on Github or the NAX website. 


\section{OPTIMIZATION}

\section{Problem statement:}

A comparison of clean and distorted flow conditions for the axisymmetric (Design A643) optimum is shown in Figure 3 . We can observe the variation of total pressure in the circumferential and radial direction, which in-turn affects the incidence on the rotor. This variation in incidence affects the rotor performance such as efficiency, stall margin and aeromechanics. Therefore, apart from providing a structural support to TCT, the IGV design is also used to reduce this nonaxisymmetry in rotor incidence (Leichtfuss et.al, 2012; Shaw et.al, 2013). The IGV exit angles are first focused for optimization. Overall 6 span locations at inlet (span 0 (hub),15,35,55,85 and $100 \%$ (tip) ) and 5 at the IGV exit plane (spans 10, 20, 45, 70, and 90\%) were identified for analysis.

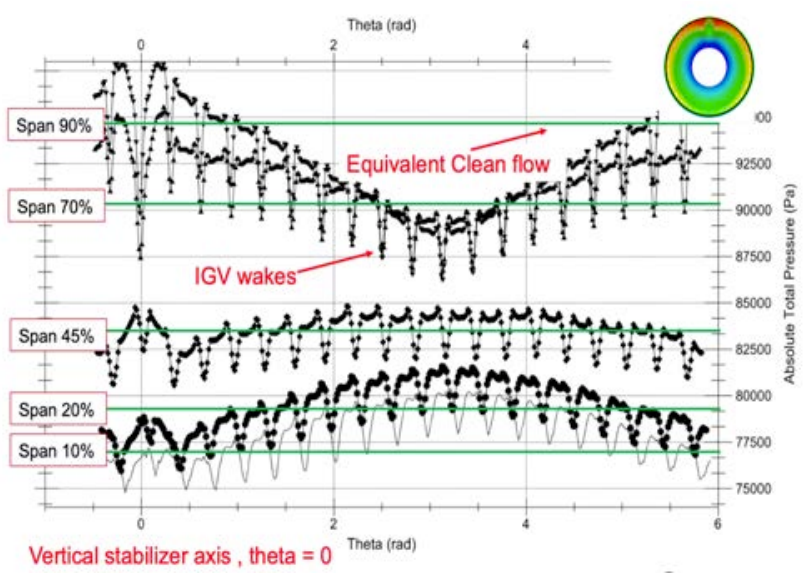

(a)

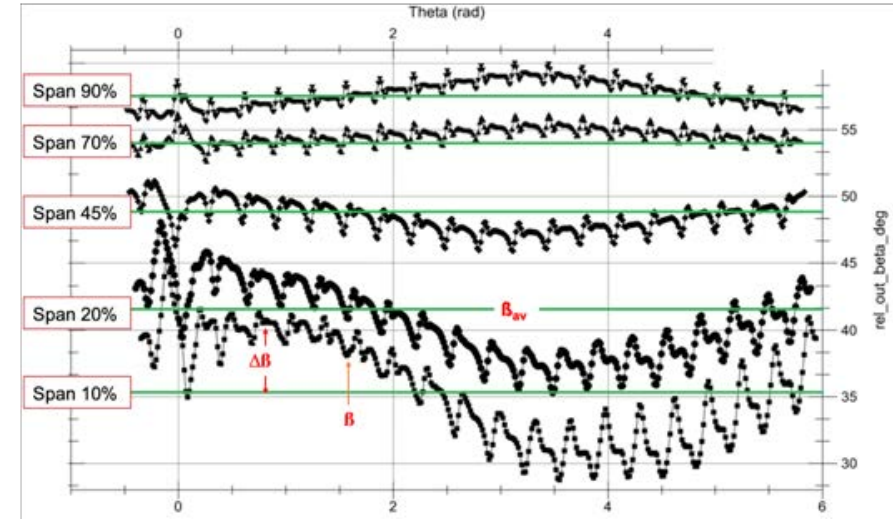

(b)

Figure 3 : Comparison of distortion profile against clean flow conditions at analysis locations. Total pressure (left) and relative flow angle (beta) (right)

Importance of relative flow angle:

To account for the effect of distortion, the relative flow angle $(\beta)$ is plotted in a rotational frame of reference at the IGV exit. This angle transformation from absolute frame $\left(\alpha=\tan ^{-1}\left(V_{\theta} / V_{z}\right)\right.$ ), into rotational frame of reference (rotor frame) is defined using Eqn. (2). This approach captures the effect of 2D circumferential distortion on the rotor (IGV exit), without carrying out actual steady CFD analysis of the IGV-rotor system. Hence, it helps in saving considerable amount of computational resources. This approach will not include the effect of a coupled flow field due to IGV-rotor interaction, but we focus on IGV design using NAX system and hence the un-coupled flow field effects are considered.

$$
\beta=\tan ^{-1}\left(\frac{\mathrm{U}-\mathrm{V}_{\theta}}{\mathrm{V}_{\mathrm{z}}}\right)
$$

As shown in Figure 3(a), total pressure distortion is severe near the tip region and comparatively moderate near the hub region. This pressure distortion results in flow field modification and hence the rotor incidence (Figure 3(b)). The variation of relative flow angle is found to be higher near the hub region which is because of variation in tangential velocity around the annulus in the boundary layer. The non-uniform distribution of flow incidence on the rotor in $2 \mathrm{D}$ is specifically detrimental at transonic flow conditions due to its sensitivity towards incidence. This analysis is very important in understanding the nature of the design problem and thus strategy to mitigate it.

\section{Process Verification: Manual design}

The process verification is an essential step and extremely helpful for demonstration purpose. For the optimization process, we use the trailing edge blade angles of IGV as design parameters. Since these parameters are aerodynamically intuitive, we have a physical understanding of these design variables and that the different spanwise locations are somewhat independent. For this case the 1 st mode of distortion profile at $15 \%$ span is considered for verification. A distribution of relative flow angle for manual design at all 5-span location is also shown against the A643 IGV design in Figure 4. There are 2 important aspects of manual design: (1) Comparison of design at 10\% span and (2) Effect of input at $10 \%$ span on other span locations $(20,45,70$ and $90 \%)$. First the magnitude and phase values for mode- 1 of the IGV exit TE (trailing edge) angle is used as NAX inputs, i.e., $a_{1}=3.982613(\mathrm{deg}), \phi_{1}=3.127447$ ( $\mathrm{rad}$ ) from a DFT of the relative-flow angle distribution. Figure 4 shows the phase shift of $\sim 180$ degree for manual design and slight increase in magnitude. The phase shift is consistent with the assignment of inputs (3.127447 rad). For magnitude, the slightly higher values are understood to be caused by the interference with the vertical stabilizer (tail) wake. 
An important aspect for a process development is to understand the correlation of different variables against the outputs. Some variation in relative flow angle is observed for $20 \%$ span in Figure $\underline{4}$ while there is no variation at rest of span locations. This change is due to the fitting of a cubic B-spline by T-Blade3, with spans 0,15 and $35 \%$ span coordinates as control points (near hub region). Thus, it can be concluded that the variation of relative flow angle is not an ideal one-toone mapping of design inputs at corresponding span location, although it's the dominant contributor.

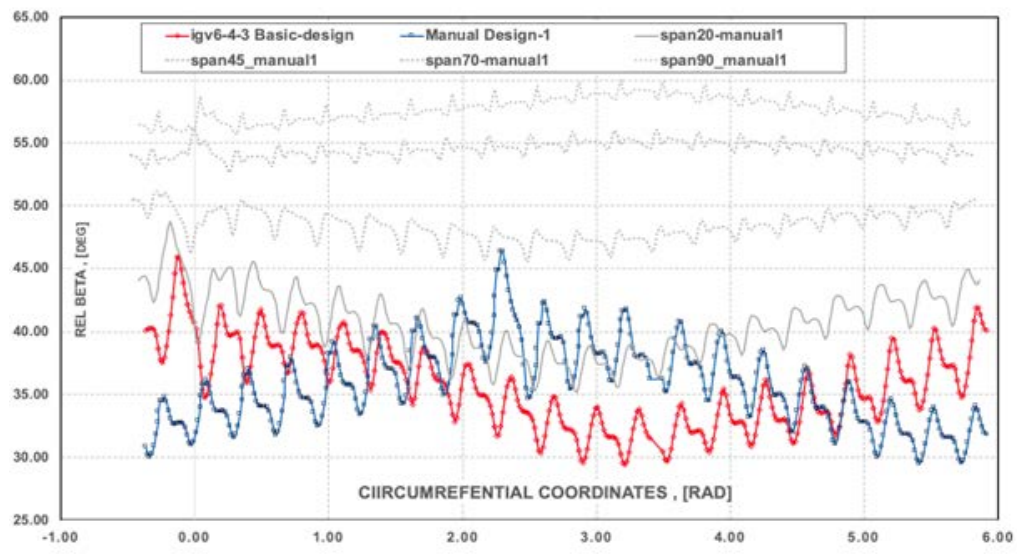

\section{Figure 4 : Comparison of relative flow angle (beta) for basic(A-643) and manual design. Coloured representation of span-10\% for manual (blue) and A-643 (red).}

\section{Optimization: Non-Linear Least Squares Methods (NL2SOL) and Genetic Algorithm (SO-GA)}

The optimization process is divided into two logical separate steps in order to achieve high methodological efficiency by exploiting the nature of problem. First, least-square method is used to is to minimize, $\Delta \beta\left(=\beta_{\theta}-\beta_{a v}\right)$, which essentially means reducing the difference between the distortion based aerodynamic performance against clean flow conditions. The angle, $\beta_{\theta}$ is the calculated angle in distorted flow and $\beta_{a v}$ is the angle corresponding to clean flow (Figure 3(b)). The NL2SOL method (Adams et al., 2020) then solves the system of equations for all 5 analysis spans at the IGV exit using only the first mode. To understand the design space, a set of 5 sub-optimizations with 150 iterations each, were carried out for each span using NAX. The overall goal of this work is to increase the propulsive efficiency of the TCT unit. Thus, the second step is to minimize circumferentially averaged loss of the IGV, $(\zeta=(\mathrm{P} 1-\mathrm{P} 2) /(\mathrm{P} 1-\mathrm{p} 2))$ (Denton 1993) as a stage performance metric using GA (Adams et al., 2020). Note that a turbine loss coefficient is used for the IGV since the IGV accelerates the flow. The optimum from NL2SOL is used as an upper limit to ensure improvement over a previous design.

An important consideration here is the sub-division of the optimization process. That is to say that the value of $\beta_{a v}$ was foreknown and the optimum is to reach this value $(\Delta \beta \rightarrow>0)$, which corresponds to a clean flow performance. There is no such expected $\zeta$ to target the loss coefficient, hence GA are best suited for this optimization to be explored.

\begin{tabular}{|c|c|c|}
\hline Method & Objective & Design Parameters \\
\hline Manual Design & Process verification & $a_{1}=3.982613(\mathrm{deg}), \phi_{1}=3.127447(\mathrm{rad})$ at $\operatorname{span} 15 \%$ \\
\hline NL2SOL & $\begin{array}{c}\operatorname{minimize} \Delta \beta \\
\left(=\beta_{\theta}-\beta_{a v}\right) \text { at } 5 \\
\text { analysis spans }-\mathrm{IGV} \text { exit }\end{array}$ & $\begin{array}{l}\text { - Iterations }=300 \text {, Central difference scheme, } 3 \text { point multi- } \\
\text { start (DOE by Latin Hyper Cube), step size } 1 . e-4 \\
\text { Inputs: } 12 \text { total variables, Magnitude and phase (1 mode) } \\
\text { of IGV TE blade angle: a1 }[0,8], \phi_{1}[-\pi, \pi] \text { each for } 6 \\
\text { spans. }\end{array}$ \\
\hline $\begin{array}{l}\text { Single Objective Genetic } \\
\text { Algorithm (SO-GA) }\end{array}$ & Minimize Loss Coeff. $(\zeta)$ & $\begin{array}{l}\text { - Iterations }=900, \text { Population size }=450, \text { Mutation }=10 \% \\
\text { - Inputs: } 16 \text { total variables, Magnitude and phase }(2 \text { mode }) \\
\text { of IGV TE blade angle for span } 0,15 \% \text { and } 1 \text { mode for } \\
35,55,85 \text { and } 100 \% \text { spans: } a_{i}[0,8], \phi_{i}[-\pi, \pi] .\end{array}$ \\
\hline
\end{tabular}




\section{DISCUSSION OF RESULTS}

\section{Relative flow angle and DFT}

A comparison of relative flow angle values for A643 design against 2 optimum is shown in Figure 5 along with their corresponding DFT results. The effect of using 2 modes at near hub span for GA against 1 mode for NL2SOL is clearly distinguishable here. The results demonstrate the multi-mode capability of NAX in blade design and provides a comparative assessment of both methods. With comparison at near hub region (spans 10 and 20\%), a significant reduction in $\Delta \beta$ is achieved for optimum designs. Using the DFT information, we can observe that although NL2SOL optimum has improved the design, the effect of using 2 modes in GA based design has also reduced the magnitude to almost half (first mode) during optimization for reduction in loss coefficient. For the optimization at span 45\%, NL2SOL demonstrates a better optimum design as compared to GA. Although, this trend is also observed at other spans (70 and $90 \%$ ), the difference in optimum is not significant. The magnitude even increases for the GA design near the tip. This variation for GA and NL2SOL optimum design can be understood in terms of their objective specifications. NL2SOL solves a system of non-linear equations for each span i.e., the specification of NL2SOL is more explicit in reduction of $\Delta \beta$. In contrast to this, GA minimizes the circumferentially averaged loss-metric calculated at IGV exit. This results in a significant reduction at hub region and less effective behaviour at tip.

\section{Loss Coefficients $(\zeta)$}

The corresponding values for loss coefficients are A643 (0.02771), NL2SOL (0.02777) and GA (0.02773). Since, no work is added in the IGV stage, the loss calculation is primarily based on the dynamic pressure head across the IGV. As discussed in (Banjac et.al, 2013), unless the blade profile has very high stagger angles, total pressure variation across the IGV is expected to be very low. As we can observe, the GA based design has been able to reduce the losses with a significant decrease in $\Delta \beta$. Because there is very small total pressure drop, the loss coefficient does not play significant role in IGV design as compared to the exit angles. The increase in losses for both optimum cases is not significant as compared to the improvements achieved over the reduction of $\Delta \beta$. Also other blade parameters such as chord, curvature and thickness have not been modified, and these would have more effect on the loss coefficient.

\section{Non-axisymmetric optimum designs}

Figure $\underline{6}$ shows a comparison of tangential component of velocity $\left(V_{\theta}\right)$ and $\beta$ at the IGV exit. These results should be referred alongside Figure 7 to understand the effect of outlet-beta angle perturbations on these quantities.
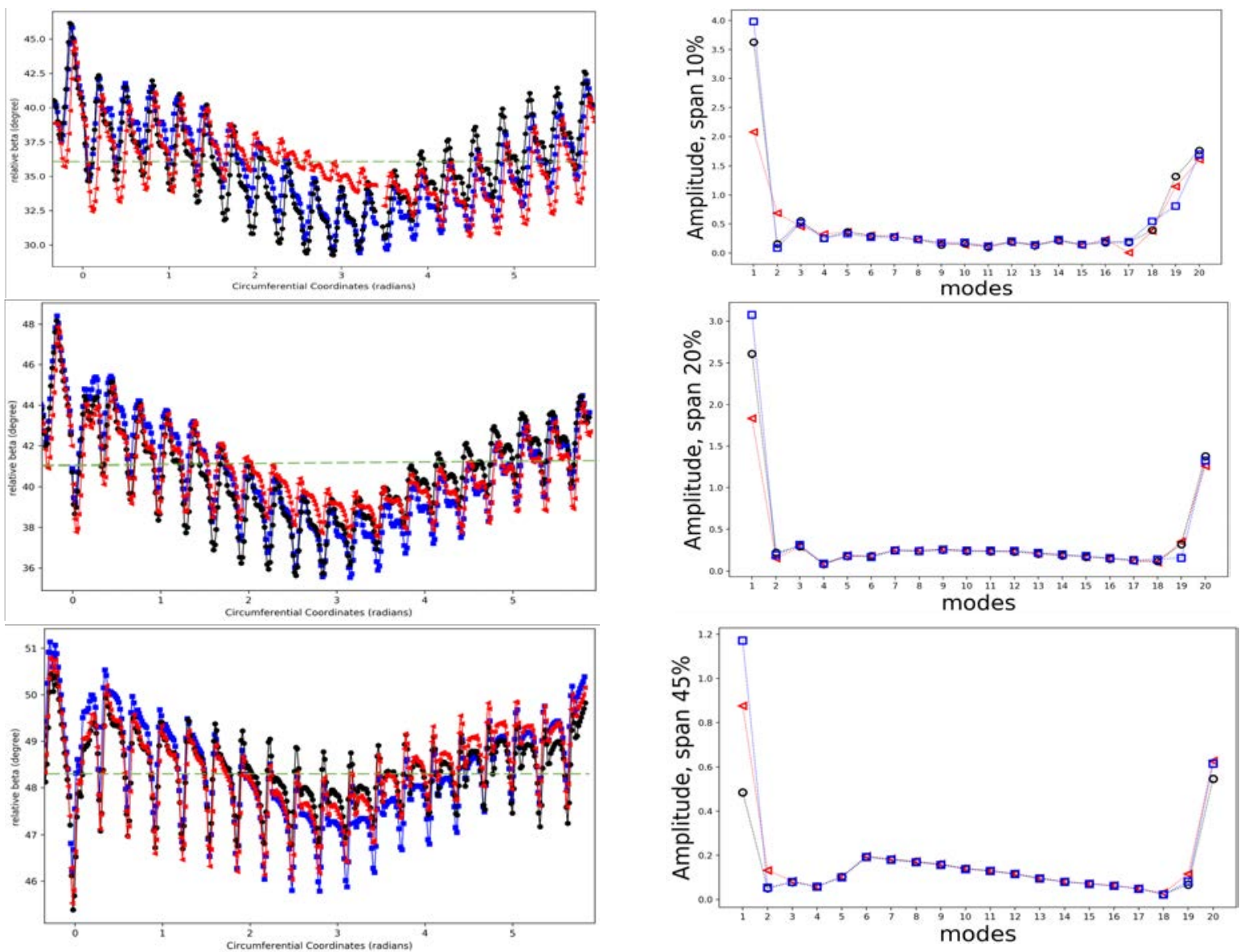

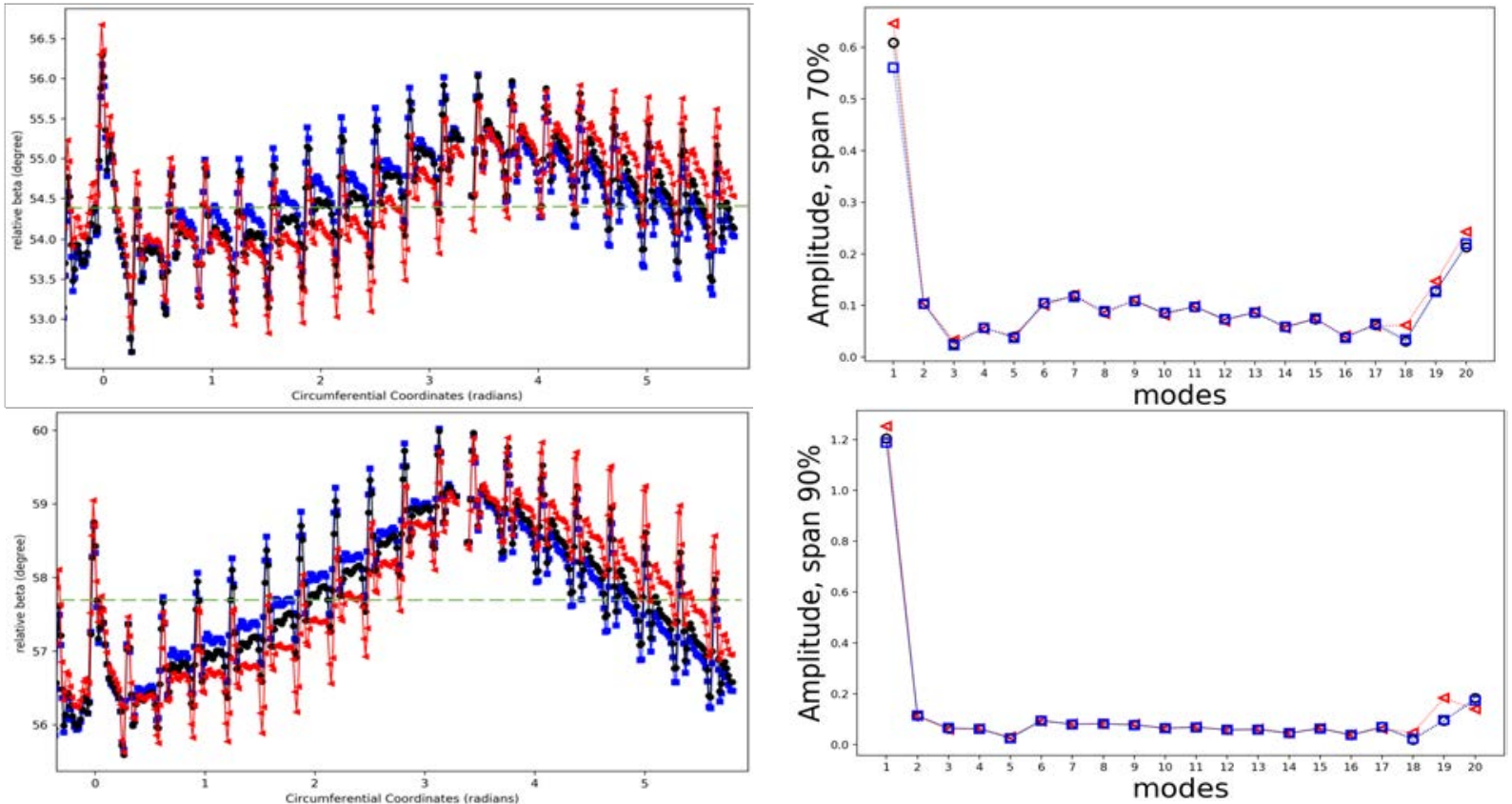

Figure 5: Comparison for relative-flow angle and corresponding DFT (magnitude) for A643 (blue), NL2SOL (black) and GA (red) optima at 5 analysis span locations. Angle corresponding to clean flow condition is shown in green.
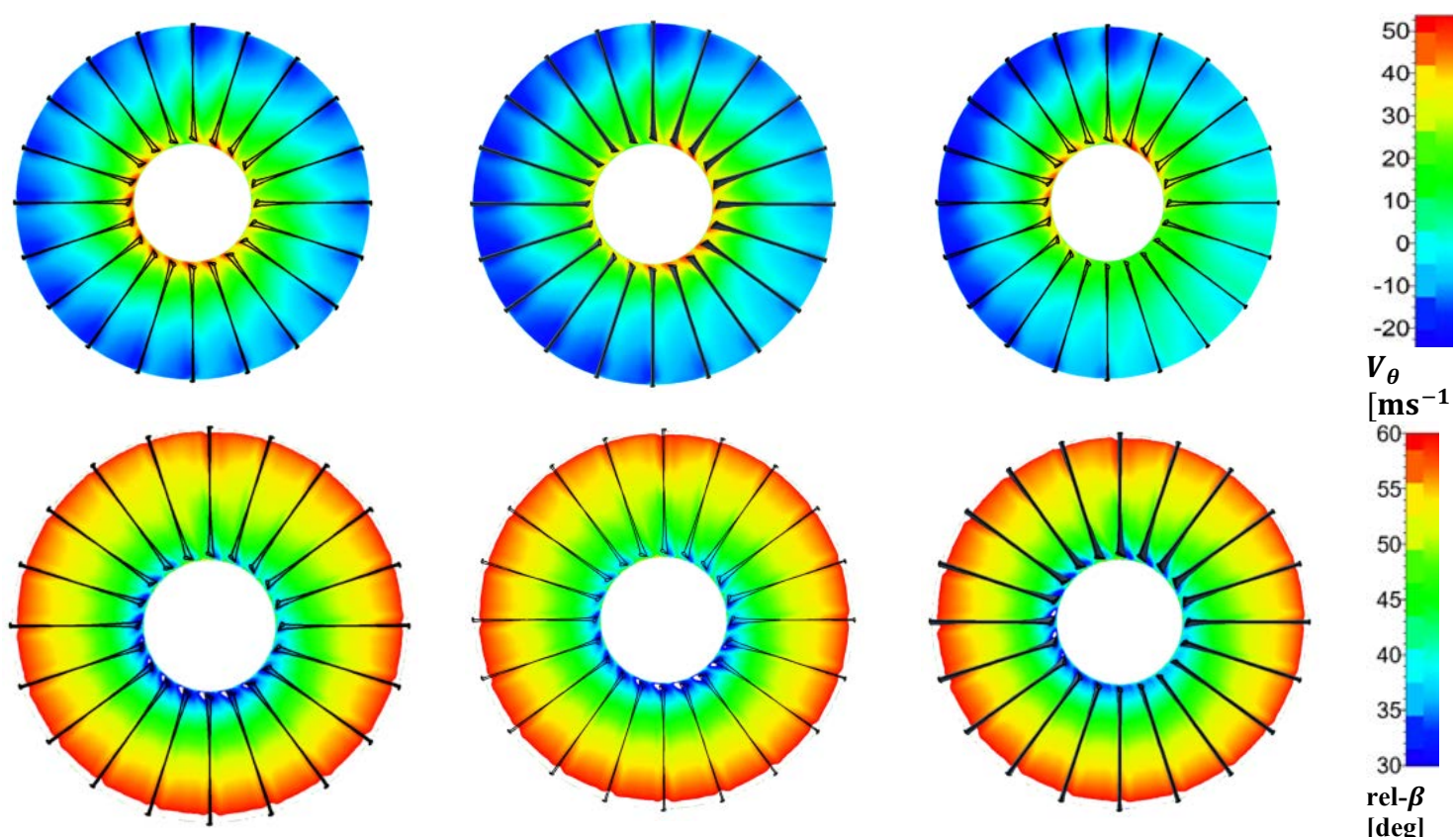

Figure 6 : Comparison of tangential velocity and relative flow angle for A643 (left), NL2SOL (middle) and GA (right) at IGV exit. Angles are measured from vertical in CW direction.
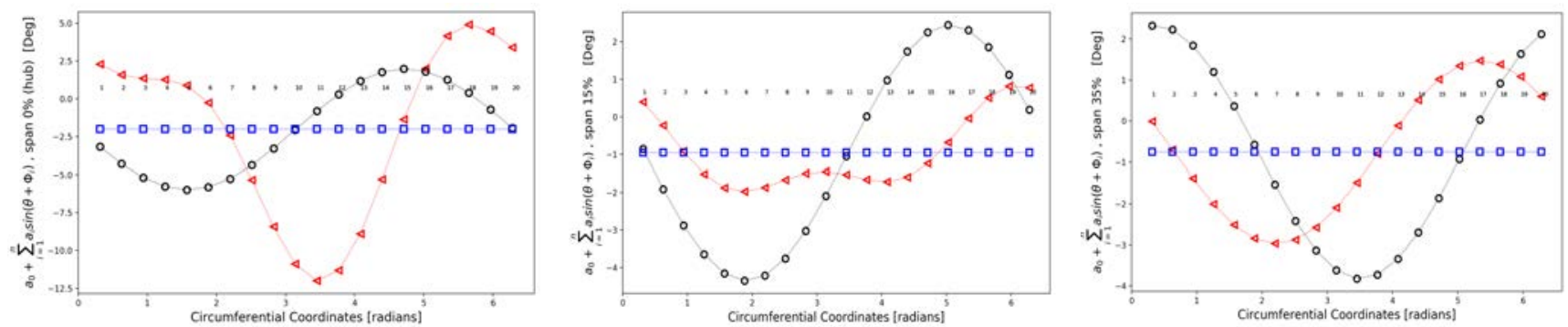

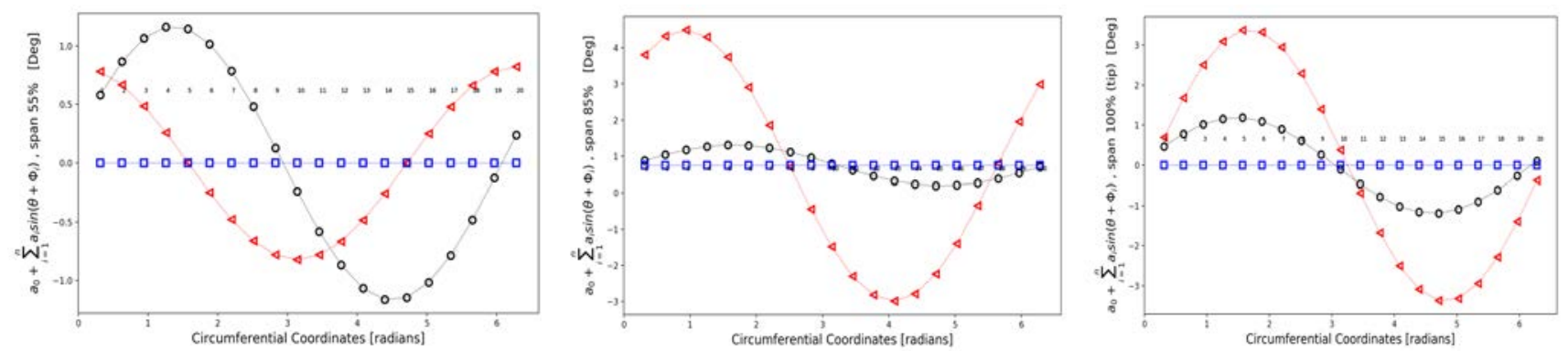

Figure 7: Comparison of IGV modification of the trailing edge angles for A643 (blue), NL2SOL (black) and $\mathrm{GA}(\mathrm{red})$ optimum.

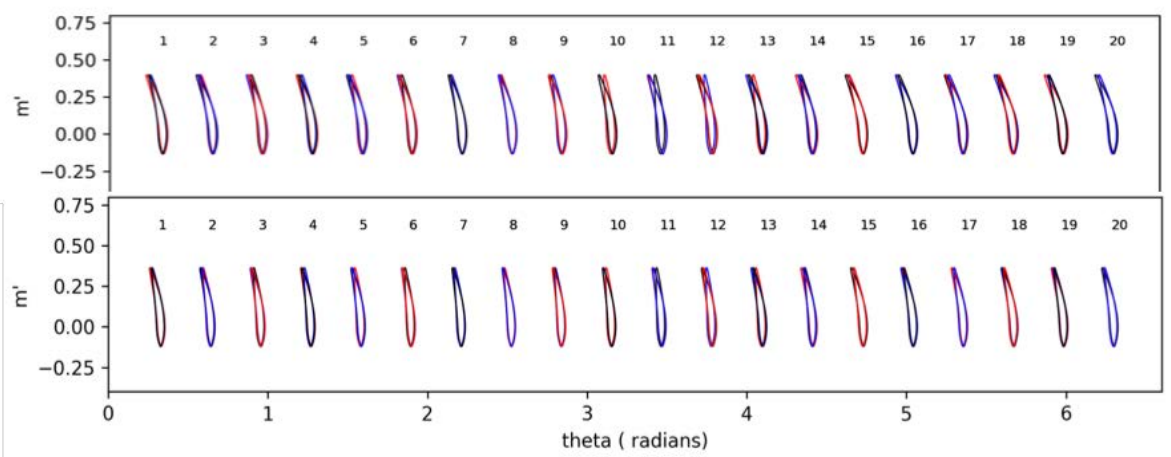

Figure 8: Comparison of m-thetas for A643 (blue), NL2SOL (black) and GA (red) optimum designs showing non-axisymmetry in blades due to outlet-beta. From top-section 1 and 3 (total 21) near hub.
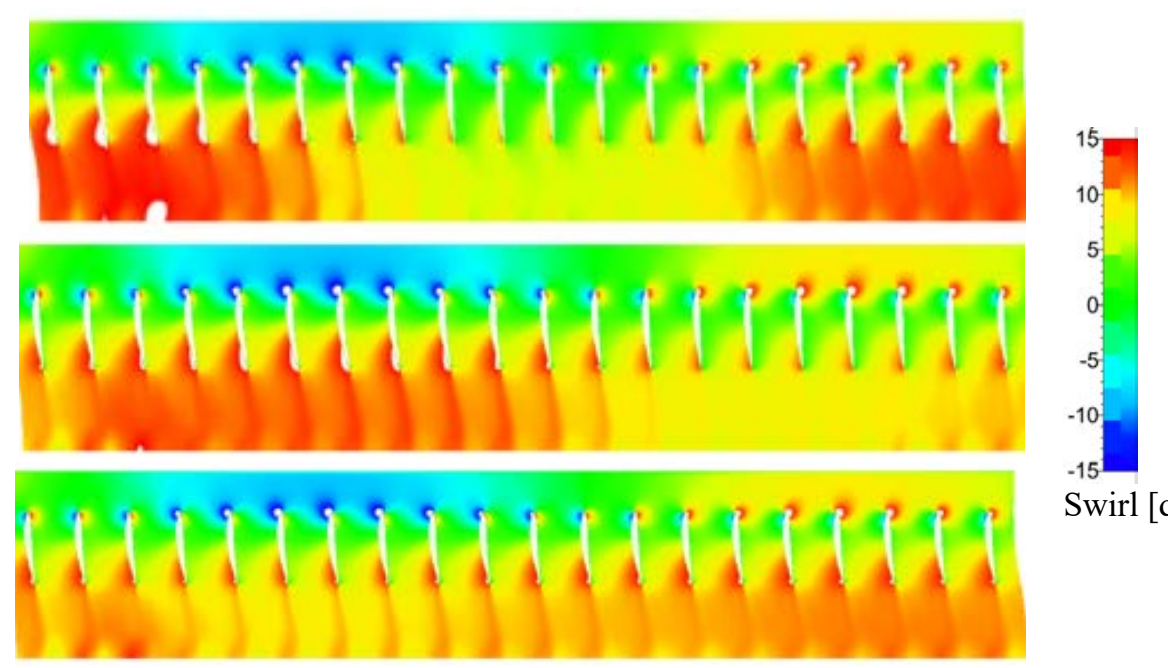

Swirl [deg]

Figure 9: Absolute swirl distribution for basic design A643 (bottom), NL2SOL -1 mode (mid) and GA -2 modes (top) optimum designs showing effect of non-axisymmetry due to outlet-beta at span $10 \%$.

These calculated perturbations are added to the axisymmetric design outlet beta angles ( $a_{0}$, (blue)) to produce nonaxisymmetric designs. The difference in total pressure and axial velocity between the 3 designs is not significant and is therefore not included in Figure 6. A very clear distinction for the modification in $V_{\theta}$, by optimizer to reduce relative flow angle variation can be observed here. Although, the optimum designs result in more uniform distribution of $V_{\theta}$, the approach by both optimizations is very different. With reference to angle distribution (Figure $\underline{8}$ ) of NL2SOL design, the perturbations are phase shifted for span $0,15 \%$ span as compared to mid span to tip. This re-distributes the flow to accelerate in sector of $\theta=0-180 \mathrm{deg}$ and reverses at the tip region in sector of $\theta=180-360 \mathrm{deg}$. Similarly, the perturbations in GA based design are in-phase throughout from hub to tip. This results in acceleration of flow at hub region and deceleration at tip region. The primary reason of GA being very effective in reducing the relative flow angle near hub region can be understood by the nature of relative flow angle distribution from hub to tip (refer Figure $\underline{3}$ ). Even with single mode at hub region, the perturbation of out-beta (around annulus) follows the pattern of relative flow angle. However, since GA produces a similar perturbation pattern from hub to tip, this is not suited for tip regions, as seen for span 45, 70 and 90\% in Figure 3. Since, NL2SOL method can explicitly target the individual span locations, the 
perturbation pattern for NL2SOL optimum is different for hub and mid-tip region. This advantage of using NL2SOL over GA is evident from DFT (Figure 7), where it shows to be more effective from midspan to tip region.

The combined effect of flow field modifications by non-axisymmetric optimum designs is shown on relative flow angle distribution around the annulus. With comparison to the A643 design, we can observe a shift for relative flow angle in counter-clockwise (CCW) direction for NL2SOL and in clockwise (CW) direction for GA. This behavior is consistent with their comparative variation as shown in Figure $\underline{6}$ for near hub spans (10 and 20\%). Also, the effect for using the 2nd mode is seen with GA design resulting in more uniform distribution of relative flow angle at hub region. A similar distribution of relative flow angle can be seen for A643 and NL2SOL design, with a vertical symmetry near tip region. However, GA optimum design tends to shift this symmetry plane CW direction by about $\theta=30-45 \mathrm{deg}$. This observation is consistent with the perturbations shown in Figure $\underline{8}$. At near tip region, NL2SOL design approaches to A643 design, whereas GA optimum has results in departure from A643 angle distribution. Comparison of $m^{\prime}-\theta$ plots near hub is shown in Figure 9, by superimposing the optimum designs with the basic design (A643). The difference is more identifiable for GA non-axisymmetric design.

A comparison of absolute swirl is presented in Figure 9 between axisymmetric and non-axisymmetric designs (using different modes) at span $10 \%$ locations. The difference in swirl is not much noticeable for mid-span locations, but very evident near hub. These results correspond to the non-uniformity in total pressure, swirl and meridional flow, but swirl non uniformity plays a significant role in flow redistribution. It can be observed that each blade experiences a combination of counter-and-co swirl across pressure and suction surfaces along blade length. We can also observe different IGV blades with decreasing positive swirl (co-swirl) and increasing negative swirl (counter-swirl) along with corresponding difference (subtle) at trailing edge swirl. The reason for this variation is total pressure non-uniformity in flow (passage), which effects the tangential velocity component and further modifies the effect of non-uniform swirl. Also, the distribution of swirl near TE is not completely anti-symmetrical about no-swirl (zero) line. Thus, we can conclude the effect of distortion tends to increase the positive swirl at LE which is countered at TE with negative swirl.

\section{SUMMARY AND CONCLUSIONS}

This paper presents the optimization of an IGV row operating in the non-axisymmetric, BLI conditions of a 1.5 stage transonic TCT unit. The optimization was completed with the open source blade design system, NAX. The key objective is to reduce the incidence on the downstream rotor to help mitigate aero-mechanical issues. A novel technique of using relative-flow angle calculated at IGV exit transformed into rotor frame of reference is used to reduce the computational resources and optimize the IGV design. This design-optimization process explores the best features of gradient based and Evolutionary Algorithms. Further, two optimum designs are discussed in comparison to the basic design for its effect on physical properties. Nearly $50 \%$ reduction in harmonic content of relative flow angle distortion is achieved, at the hub which lies in the ingested boundary layer of air-vehicle. This comprehensive design-optimization process can be used as reference by designers of BLI based designs. The main features of this work can be categorized as:

- Multi-fidelity Design system: Preliminary design capabilities are used to define 1D radial distribution of properties like angular momentum, blockage and losses as design inputs. This step helps in generating a distortion tolerant design at the through-flow calculations stage.

- Non-axisymmetric design capability: The automated design open source code, NAX is described in detail with special focus on its parametrization scheme and integration into overall design framework. Further, its suitability for application to parametrize blade design parameter in $r, \theta$ design space using multi-mode capability is demonstrated by the manual verification and 2 optimization methods.

- Non-axisymmetric Optimization: The optimization process is discussed by its sub-division into 2 separate design-optimization tasks. As shown in Figure 5 , generally the gradient based NL2SOL algorithm proves better for local objectives like span-specific relative-flow angle, while GA is best suited for minimization of overall stage loss metric (span 45\%). A departure in this general trend is observed with optima at span $70 \%$ which is caused by possible overshoot of NL2SOL and reflects to the requirement of reduced step size from current value. Although the global step size was chosen to balance accuracy and computational requirements for overall optimization process.

- Prospective work: A multi-row TCT optimization using IGV-Rotor configuration with modification in LE/TE and aerodynamic parameters like Lean/sweep to increase the off-design performance of TCT design.

\section{ACKNOWLEDGEMENTS}

This work was supported by NASA Glenn Research Centre via Vantage Partners, LLC Grant GESS3 GL/TL W141.

\section{REFERENCES}

Adams, B.M., Bohnhoff, W.J., Dalbey, K., Ebeida, M.S., Eddy, J.P., Eldred, M.S., Hooper, R., Hough, P.D., Hu, K., Jakeman, J.D. and Khalil, M., 2020. Dakota A Multilevel Parallel Object-Oriented Framework for Design Optimization Parameter Estimation Uncertainty Quantification and Sensitivity Analysis: Version 6.12 User Manual (No. SAND2020- 
5001). Sandia National Lab.(SNL-NM), Albuquerque, NM (United States); Sandia National Lab.(SNL-CA), Livermore, CA (United States).

Armstrong, M.J., Ross, C.A., Blackwelder, M.J. and Rajashekara, K., 2012. Trade studies for NASA N3-X turboelectric distributed propulsion system electrical power system architecture. SAE International Journal of Aerospace, 5(2012-012163), pp.325-336.

Banjac, M., Petrovic, M.V. and Wiedermann, A., 2014. A new loss and deviation model for axial compressor inlet guide vanes. Journal of Turbomachinery, 136(7).

Denton, J.D., 1993. The 1993 IGTI scholar lecture: Loss mechanisms in turbomachines.

Felder, J., Kim, H. and Brown, G., 2009, January. Turboelectric distributed propulsion engine cycle analysis for hybridwing-body aircraft. In 47th AIAA aerospace sciences meeting including the new horizons forum and aerospace exposition (p. 1132).

Gorrell, S.E., Copenhaver, W.W. and Chriss, R.M., 2001. Upstream wake influences on the measured performance of a transonic compressor stage. Journal of Propulsion and Power, 17(1), pp.43-48.

Gorrell, S.E., Car, D., Puterbaugh, S.L., Estevadeordal, J. and Okiishi, T.H., 2006. An investigation of wake-shock interactions in a transonic compressor with digital particle image velocimetry and time-accurate computational fluid dynamics.

Greitzer, E.M. and Griswol, H.R., 1976. Compressor-diffuser interaction with circumferential flow distortion. Journal of Mechanical Engineering Science, 18(1), pp.25-38..

Greitzer, E.M., Mazzawy, R.S. and Fulkerson, D.A., 1978. Flow field coupling between compression system components in asymmetric flow.

Gunn, E.J. and Hall, C.A., 2017, June. Non-axisymmetric stator design for boundary layer ingesting fans. In Turbo Expo: Power for Land, Sea, and Air (Vol. 50770, p. V001T01A003). American Society of Mechanical Engineers.

Kim, H.D., Brown, G.V. and Felder, J.L., 2008. Distributed turboelectric propulsion for hybrid wing body aircraft.

Kumar, S., Turner, M.G., Siddappaji, K. and Celestina, M., 2018. Aerodynamic Design System for Non-Axisymmetric Boundary Layer Ingestion Fans. In ASME Turbo Expo 2018: Turbomachinery Technical Conference and Exposition. American Society of Mechanical Engineers Digital Collection.

Leichtfuss, S., Biela, C., Schiffer, H.P. and Heinichen, F., 2012, June. Influence of inlet guide vane wakes on the passage flow in a transonic axial compressor. In ASME Turbo Expo 2012: Turbine Technical Conference and Exposition (pp. 2721-2729). American Society of Mechanical Engineers Digital Collection.

Longley, J.P. and Greitzer, E.M., 1992. Inlet distortion effects in aircraft propulsion system integration.

Mandal. P, Holder J, Turner M.G. and Celestina M.L. "Draft:Design and Optimization of Boudnary Layer Ingesting Propulsor", In ASME Turbo Expo2020: Gas Turbine Technical Conference and Exposition -15603, In-press.

Numeca Fine/Turbo V12.2. n.d. https://www.numeca.com/en_us/product/fineturbo.

Sharma. M, Dannenhoffer III. J.F, Holder J and Turner M.G. Draft: A 3D turbomachinery design system integrating Physics-based Parametrization with Engineering Sketch Pad", In ASME Turbo Expo2020: Gas Turbine Technical Conference and Exposition -15943, In-press.

Siddappaji, K., Turner, M.G. and Merchant, A., 2012, June. General capability of parametric 3d blade design tool for turbomachinery. In ASME Turbo Expo 2012: Turbine Technical Conference and Exposition (pp. 2331-2344). American Society of Mechanical Engineers Digital Collection.

Singh, S.K., 2017. A novel concept for non-linear multidisciplinary aerodynamic design optimization. Aerospace Science and Technology, 70, pp.626-635.

Shaw, M.J., Hield, P. and Tucker, P.G., 2014. The effect of inlet guide vanes on inlet flow distortion transfer and transonic fan stability. Journal of Turbomachinery, 136(2).

Turner, M.G., Merchant, A. and Bruna, D., 2011. A turbomachinery design tool for teaching design concepts for axialflow fans, compressors, and turbines. Journal of turbomachinery, 133(3). 